\title{
The Jacobian Conjecture in case of "non-negative coefficients"
}

\author{
by LUDWIK M. DRużKowski (Kraków)
}

Abstract. It is known that it is sufficient to consider in the Jacobian Conjecture only polynomial mappings of the form

$$
F\left(x_{1}, \ldots, x_{n}\right)=x-H(x):=\left(x_{1}-H_{1}\left(x_{1}, \ldots, x_{n}\right), \ldots, x_{n}-H_{n}\left(x_{1}, \ldots, x_{n}\right)\right),
$$

where $H_{j}$ are homogeneous polynomials of degree 3 with real coefficients (or $H_{j}=0$ ), $j=1, \ldots, n$ and $H^{\prime}(x)$ is a nilpotent matrix for each $x=\left(x_{1}, \ldots, x_{n}\right) \in \mathbb{R}^{n}$.

We give another proof of Yu's theorem that in the case of non-negative coefficients of $H$ the mapping $F$ is a polynomial automorphism, and we moreover prove that in that case

$$
\operatorname{deg} F^{-1} \leq(\operatorname{deg} F)^{\text {ind } F-1}, \quad \text { where } \quad \text { ind } F:=\max \left\{\operatorname{ind} H^{\prime}(x): x \in \mathbb{R}^{n}\right\} .
$$

Note that the above inequality is not true when the coefficients of $H$ are arbitrary real numbers; cf. [E3].

1. Formulation of the Jacobian Conjecture. Let $\mathbb{K}$ denote either $\mathbb{C}$ or $\mathbb{R}$. Define

$$
\begin{aligned}
\mathcal{P}\left(\mathbb{K}^{n}\right):=\left\{F=\left(F_{1}, \ldots, F_{n}\right): \mathbb{K}^{n} \rightarrow\right. & \mathbb{K}^{n}: \\
& \left.F_{j} \in \mathbb{K}\left[X_{1}, \ldots, X_{n}\right], j=1, \ldots, n\right\} .
\end{aligned}
$$

In other words, $\mathcal{P}\left(\mathbb{K}^{n}\right)$ is the set of polynomial transformations of $\mathbb{K}^{n}$. We set

$$
\operatorname{Jac} F:=\operatorname{det}\left[\frac{\partial F_{i}}{\partial x_{j}}: i, j=1, \ldots, n\right] .
$$

Now we recall the $n$-dimensional Jacobian Conjecture (briefly $(\mathrm{JC})_{n}$ ) for $n \geq 2$ :

1991 Mathematics Subject Classification: Primary 14E09.

Key words and phrases: polynomial automorphisms, nilpotent matrix, Jacobian Conjecture.

Partially supported by KBN Grant no. 2 P03A 06108. 
$(\mathrm{JC})_{n} \quad\left[F \in \mathcal{P}\left(\mathbb{K}^{n}\right)\right.$ and $\operatorname{Jac} F=$ const $\neq 0$ for every $\left.x \in \mathbb{K}^{n}\right]$

$$
\Rightarrow[F \text { is injective }] \text {, }
$$

and the Generalized Jacobian Conjecture ((GJC) for short):

$(J C)_{n}$ holds for every $n \geq 2$.

It is common to call the $n$-dimensional Jacobian Conjecture or Generalized Jacobian Conjecture briefly the Jacobian Conjecture, yet to avoid confusion we often use the notation introduced above throughout the paper. For survey papers and papers containing other equivalent formulations of the Jacobian Conjecture we refer the reader to e.g. [BCW], [D3], [E1], [E2], [KS], [M], $[\mathrm{MO}],[\mathrm{R}],[\mathrm{S}]$.

Note that up to this time the Jacobian Conjecture remains unsolved even if $n=2$ and the stronger conjecture in which instead of Jac $F=$ const $\neq 0$ for $F \in \mathcal{P}\left(\mathbb{R}^{n}\right)$ we put $\pm \operatorname{Jac} F>0$ is false (cf. $[\mathrm{P}]$ ).

A polynomial mapping $F \in \mathcal{P}\left(\mathbb{C}^{n}\right)$ can be treated as $\widehat{F}=(\operatorname{Re} F, \operatorname{Im} F) \in$ $\mathcal{P}\left(\mathbb{R}^{2 n}\right)$ and then Jac $\widehat{F}(x, y)=|\operatorname{Jac} F(x+i y)|^{2}$. This makes it evident that the complex $(\mathrm{JC})_{n}$ follows from the real $(\mathrm{JC})_{2 n}$, so

(GJC) for polynomial mappings with real coefficients

$\Downarrow$

(GJC) for polynomial mappings with complex coefficients.

2. Reduction of the degree. First we recall some important properties of polynomial maps.

THEOREM 2.1. (i) ([BR]) Every injective polynomial map of $\mathbb{K}^{n}$ is bijective.

(ii) ([Y], [BCW], [W]) Every injective polynomial map $F$ of $\mathbb{C}^{n}$ is a polynomial automorphism, i.e. the inverse $F^{-1}$ exists and it is a polynomial mapping.

(iii) ([BCW], [RW]) If $F$ is a polynomial automorphism of $\mathbb{K}^{n}$, then $\operatorname{deg} F^{-1} \leq(\operatorname{deg} F)^{n-1}$ and (without additional assumptions) the estimate is sharp.

Now we recall the reduction theorems that are used in the investigation of (GJC).

TheOREM 2.2 ([Y], [BCW], [D1]). If we consider the Generalized Jacobian Conjecture (i.e. the Jacobian Conjecture for every $n>1$ ), then it is sufficient to consider, for every $n>1$, only polynomial mappings of the so-called cubic homogeneous form $F=I-H$, where $I$ denotes the identity, $H=\left(H_{1}, \ldots, H_{n}\right)$ and $H_{j}: \mathbb{K}^{n} \rightarrow \mathbb{K}$ is a cubic homogeneous polynomial of degree 3 or $H_{j}=0, j=1, \ldots, n$. 
It is easy to check that the cubic homogeneous form $I-H$ is invariant under the action of the full linear group $G L_{n}(\mathbb{K})$, i.e. if $F$ has a cubic homogeneous form and $L \in G L_{n}(\mathbb{K})$, then $L \circ F \circ L^{-1}$ also has a cubic homogeneous form. Let us recall the following characterization of the condition $\operatorname{Jac}(I-H)=1$.

Proposition 2.3 ([BCW], [D1]). Let $F=I-H$ be a cubic homogeneous form. Then

$$
\text { Jac } F=1 \Leftrightarrow \text { the matrix } H^{\prime}(x) \text { is nilpotent for every } x \in \mathbb{K}^{n} \text {. }
$$

Let $\widetilde{H}$ denote the unique symmetric three-linear mapping such that $\widetilde{H}(x, x, x)=H(x), x \in \mathbb{K}^{n}$. Note that $H^{\prime}(x)=3 \widetilde{H}(x, x, \cdot)$. Hence, if $\operatorname{Jac}(I-H)=1$, then by Proposition 2.3 the matrix $\widetilde{H}(x, x, \cdot)=\frac{1}{3} H^{\prime}(x)$ is nilpotent for each $x \in \mathbb{K}^{n}$. Therefore, for every $x \in \mathbb{K}^{n}$ there exists the index of nilpotency of the matrix $\left[H^{\prime}(x)\right]$, i.e. there exists a natural number $p(x)$ such that $\left[H^{\prime}(x)\right]^{p(x)}=0$ and $\left[H^{\prime}(x)\right]^{p(x)-1} \neq 0$. It is evident that

$$
1 \leq p(x) \leq 1+\operatorname{rank} H^{\prime}(x) \leq n \quad \text { for every } x \in \mathbb{K}^{n} .
$$

We define the index of nilpotency of the mapping $F=I-H$ to be the number

$$
\text { ind } F:=\sup \left\{p(x) \in \mathbb{N}:\left[H^{\prime}(x)\right]^{p(x)}=0,\left[H^{\prime}(x)\right]^{p(x)-1} \neq 0, x \in \mathbb{K}^{n}\right\} .
$$

We are able to reduce the verification of the Generalized Jacobian Conjecture to the investigation of polynomial mappings of the so-called cubic linear form, namely

THEOREM 2.4 ([D1], [D2]). In order to verify (GJC) it is sufficient to check it (for every $n>1$ ) only for polynomial mappings $F=\left(F_{1}, \ldots, F_{n}\right)$ of cubic linear form, i.e.

$$
F(x)=\left(\begin{array}{c}
x_{1} \pm\left(a_{1} x\right)^{3} \\
x_{2} \pm\left(a_{2} x\right)^{3} \\
\ldots \ldots \ldots \ldots \\
x_{n} \pm\left(a_{n} x\right)^{3}
\end{array}\right)
$$

where $x \in \mathbb{K}^{n}, a_{j}=\left(a_{j}^{1}, \ldots, a_{j}^{n}\right) \in \mathbb{K}^{n}, a_{j} x:=a_{j}^{1} x_{1}+\ldots+a_{j}^{n} x_{n}, j=$ $1, \ldots, n$, having an additional nilpotent property $(\mathrm{N})$ of the matrix $A:=$ $\left[a_{j}^{i}: i, j=1, \ldots, n\right]$ :

(N) there exists $c \in \mathbb{K}^{n}$ such that

$$
A=A_{c}:= \pm\left(\begin{array}{ccc}
\left(a_{1} c\right)^{2} a_{1}^{1} & \ldots & \left(a_{1} c\right)^{2} a_{1}^{n} \\
\ldots \ldots \ldots & \ldots & \ldots \ldots \ldots \\
\left(a_{n} c\right)^{2} a_{n}^{1} & \ldots & \left(a_{n} c\right)^{2} a_{n}^{n}
\end{array}\right) \quad \text { and } \quad \text { ind } A=\operatorname{ind} F
$$

3. Inversion formulas. Let us recall a simple inverse formula for a mapping of cubic homogeneous form. 
TheOREm 3.1 ([DR]). Let $F=I-H$, where $H$ is a homogeneous polynomial mapping of $\mathbb{C}^{n}$ of degree 3 , let $\widetilde{H}$ denote the unique symmetric three-linear mapping such that $\widetilde{H}(x, x, x)=H(x)$ and let

$$
G=\sum_{j=1}^{\infty} G^{[j]}, \quad \text { where } G^{[j]} \text { are homogeneous of degree } j,
$$

be the formal inverse of $F$. Then

$$
\begin{aligned}
G^{[1]} & =I, \\
G^{[2 k+1]} & =\sum_{p+q+r=k-1} \widetilde{H}\left(G^{[2 p+1]}, G^{[2 q+1]}, G^{[2 r+1]}\right), \quad k=1,2,3, \ldots, \\
G^{[2 k]} & =0, \quad k=1,2,3, \ldots
\end{aligned}
$$

In particular,

$$
\begin{aligned}
G^{[3]} & =H, \\
G^{[5]} & =3 \widetilde{H}(I, I, H), \\
G^{[7]} & =9 \widetilde{H}(I, I, \widetilde{H}(I, I, H))+3 \widetilde{H}(I, H, H), \\
G^{[9]} & =27 \widetilde{H}(I, I, \widetilde{H}(I, I, H(I, I, H))+9 \widetilde{H}(I, I, \widetilde{H}(I, I, H)) \\
& =18 \widetilde{H}(I, I, \widetilde{H}(I, I, H))+\widetilde{H}(H, H, H) .
\end{aligned}
$$

Moreover, it was conjectured in $[\mathrm{DR}]$ that

$$
\operatorname{deg} F^{-1} \leq(\operatorname{deg} F)^{\text {ind } F-1}, \quad F=I-H,
$$

and the estimate was checked for ind $F=2$ and $n$.

Now we recall the following Yu's theorem.

TheOrem $3.2([\mathrm{Yu}])$. Let $F=I-N: \mathbb{C}^{n} \rightarrow \mathbb{C}^{n}$ be a polynomial map with real coefficients such that $\operatorname{Jac} F=1$, ord $N \geq 2(N$ is not necessarily homogeneous) and every coefficient of $N$ is non-negative. Then $F$ is a stably tame automorphism. That is, $F$ is a polynomial automorphism and there exists an integer $m \geq 0$ such that $\widehat{F}=\left(F, X_{n+1}, \ldots, X_{n+m}\right): \mathbb{C}^{m} \rightarrow \mathbb{C}^{m}$ is a tame automorphism (i.e. $\widehat{F}$ is a composition of linear and triangular polynomial automorphisms).

To investigate the Generalized Jacobian Conjecture it is sufficient (due to Theorem 2.2) to consider only the polynomial mappings of cubic homogeneous form $F=I-H$, where $H$ is homogeneous of degree 3 . Using Theorem 3.1 we will prove the following version of Yu's Theorem 3.2 with the estimate of the degree of the inverse conjectured in [DR].

THEOREM 3.3. If $F=I-H: \mathbb{R}^{n} \rightarrow \mathbb{R}^{n}$, Jac $F=1$ and all coefficients of the cubic homogeneous polynomial mapping $H$ are non-negative, 
then $F$ is invertible and the degree of its inverse $F^{-1}$ is not greater than $(\operatorname{deg} F)^{\text {ind } F-1}$.

The above theorem was announced in [D3] and proved in the case of ind $F \leq 3$. In the proof given below we avoid the arguments from matrix theory used in $[\mathrm{Yu}]$ and we show that Theorem 3.3 is a direct and natural consequence of the inverse formula given in Theorem 3.1.

Proof. Notation: If $\Phi=\left(\Phi_{1}, \ldots, \Phi_{s}\right): \mathbb{R}^{n} \rightarrow \mathbb{R}^{s}$ is a formal power series mapping, then, for $j=1, \ldots, s$,

(0) $\Phi \geq 0 \Leftrightarrow$ all coefficients of any power series $\Phi_{j}$ are non-negative.

By Theorem 3.1 the mapping $G$ (which is a local inverse of $F=I-H$ ) is of the form

$$
G=\sum_{j=0}^{\infty} G^{[2 j+1]}, \quad \text { where } G^{[k]} \text { are homogeneous of degree } k,
$$

with

$$
G^{[1]}=I,
$$

(2) $\quad G^{[2 k+1]}=\sum_{p+q+r=k-1} \tilde{H}\left(G^{[2 p+1]}, G^{[2 q+1]}, G^{[2 r+1]}\right), \quad k=1,2,3, \ldots$,

$\widetilde{H}$ being the unique symmetric three-linear mapping such that $\widetilde{H}(x, x, x)=$ $H(x)$.

Since $F(x)=x-H(x)$ and ind $F=p, p \leq n$, we have

$$
\begin{aligned}
F^{\prime}(x) & =I-H^{\prime}(x)=I-3 \widetilde{H}(x, x, \cdot), \\
F^{\prime \prime}(x) & =-H^{\prime \prime}(x)=-6 \widetilde{H}(x, \cdot, \cdot), \\
F^{\prime \prime \prime}(x) & =-H^{\prime \prime \prime}(x)=-6 \widetilde{H}, \\
{\left[F^{\prime}(x)\right]^{-1} } & =\sum_{i=0}^{p-1}\left[H^{\prime}(x)\right]^{i}=\sum_{i=0}^{p-1} 3^{i}[\widetilde{H}(x, x, \cdot)]^{i},
\end{aligned}
$$

where

$$
\begin{aligned}
{\left[H^{\prime}(x)\right]^{0} } & =I, \\
{\left[H^{\prime}(x)\right]^{i+1} } & =H^{\prime}(x) \circ\left[H^{\prime}(x)\right]^{i}=\left[H^{\prime}(x)\right]^{i} \circ H^{\prime}(x), \\
{[\widetilde{H}(x, x, \cdot)]^{0} } & =I, \\
{[\widetilde{H}(x, x, \cdot)]^{i+1} } & =\widetilde{H}(x, x, \cdot) \circ[\widetilde{H}(x, x, \cdot)]^{i}=[\widetilde{H}(x, x, \cdot)]^{i} \circ \widetilde{H}(x, x, \cdot) .
\end{aligned}
$$

Since $3\left[H^{\prime}(x)\right]^{i}[H(x)]=\left[H^{\prime}(x)\right]^{i+1}(x)$, by (3) we get the equality 
(4) $\left[F^{\prime}(x)\right]^{-1}[F(x)]=x-H(x)+\sum_{i=1}^{p-1}\left[H^{\prime}(x)\right]^{i}[x-H(x)]$

$$
\begin{aligned}
& =x-H(x)+\sum_{i=1}^{p-1}\left\{\left[H^{\prime}(x)\right]^{i}(x)-\frac{1}{3}\left[H^{\prime}(x)\right]^{i+1}(x)\right\} \\
& =x+2 H(x)+\frac{2}{3} \sum_{i=2}^{p-1}\left[H^{\prime}(x)\right]^{i}(x) \\
& =x+2 \sum_{i=1}^{p-1} 3^{i-1}[\widetilde{H}(x, x, \cdot)]^{i-1}[H(x)] .
\end{aligned}
$$

From (1) we obtain

(5)

$$
x=\sum_{j=0}^{\infty} G^{[2 j+1]}[x-H(x)], \quad \text { i.e. } \quad x=\left.\sum_{j=0}^{\infty} G^{[2 j+1]}(y)\right|_{y=x-H(x)}
$$

and by the above equality we have

(6) $H(x)=\sum_{j=1}^{\infty} G^{[2 j+1]}[x-H(x)]$, i.e. $H(x)=\left.\sum_{j=1}^{\infty} G^{[2 j+1]}(y)\right|_{y=x-H(x)}$.

Differentiating (5) and then taking the value of the differential at the vector $x-H(x)$ we derive the equality

$$
\sum_{j=0}^{\infty}(2 j+1) G^{[2 j+1]}[x-H(x)]=\left[F^{\prime}(x)\right]^{-1}[x-H(x)] .
$$

By (4) and (7) we get

(8) $\sum_{j=1}^{\infty}(2 j+1) G^{[2 j+1]}[x-H(x)]=H(x)+2 \sum_{i=1}^{p-1} 3^{i-1}[\widetilde{H}(x, x, \cdot)]^{i-1}[H(x)]$.

Due to (5), (6) and (8) we have

$$
\begin{aligned}
\sum_{j=1}^{\infty}(2 j & +1) G^{[2 j+1]}[x-H(x)] \\
= & 3 H(x) \\
& +2 \sum_{i=2}^{p-1} 3^{i-1}\left\{\widetilde{H}\left[\sum_{j=0}^{\infty} G^{[2 j+1]}(x-H(x)), \sum_{j=0}^{\infty} G^{[2 j+1]}(x-H(x)), \cdot\right]\right\}^{i-1} \\
& \times\left[\sum_{j=1}^{\infty} G^{[2 j+1]}(x-H(x))\right],
\end{aligned}
$$


so putting $y=x-H(x)$ in the above equality and using (6) we obtain

(9) $\sum_{j=1}^{\infty}(2 j+1) G^{[2 j+1]}(y)=3 \sum_{j=1}^{\infty} G^{[2 j+1]}(y)$
$+2 \sum_{i=2}^{p-1} 3^{i-1}\left\{\widetilde{H}\left[\sum_{j=0}^{\infty} G^{[2 j+1]}(y), \sum_{j=0}^{\infty} G^{[2 j+1]}(y), \cdot\right]\right\}^{i-1}\left[\sum_{j=0}^{\infty} G^{[2 j+1]}(y)-y\right]$.

Note that

$$
\begin{gathered}
G^{[2 j+1]} \geq 0, \quad \widetilde{H}\left(G^{[2 j+1]}, G^{[2 k+1]}, G^{[2 m+1]}\right) \geq 0, \\
{\left[\widetilde{H}\left(G^{[2 j+1]}, G^{[2 k+1]}, \cdot\right)\right]^{r} \geq 0}
\end{gathered}
$$

for any $j, k, m, r \in \mathbb{N}$.

According to (10) our strategy is the following: write all formal series mappings with non-negative coefficients (beginning with some order) on one side of the equality and leave 0 on the other side of this equality.

From (9), (5) and (6) we derive

$$
\begin{aligned}
& \sum_{j=1}^{\infty}(2 j+1-3) G^{[2 j+1]}(y) \\
& +\sum_{i=2}^{p-1} \frac{3^{i-1}}{2}\left\{\widetilde{H}\left[\sum_{j=0}^{\infty} G^{[2 j+1]}(y), \sum_{j=0}^{\infty} G^{[2 j+1]}(y), \cdot\right]\right\}^{i-1}(y) \\
= & 2 \sum_{i=2}^{p-1} 3^{i-1}\left\{\widetilde{H}\left[\sum_{j=0}^{\infty} G^{[2 j+1]}(y), \sum_{j=0}^{\infty} G^{[2 j+1]}(y), \cdot\right]\right\}^{i-2}\left[\sum_{k=1}^{\infty} G^{[2 k+1]}(y)\right],
\end{aligned}
$$

since $G^{[2 k+1]}=\sum_{p+q+r=k-1} \widetilde{H}\left(G^{[2 p+1]}, G^{[2 q+1]}, G^{[2 r+1]}\right)$. The equality (11) can be written in the form

$$
\begin{aligned}
& \text { (12) } \sum_{j=01}^{\infty}(2 j+1-3) G^{[2 j+1]}(y)+R_{1}(y) \\
& =2 \sum_{i=2}^{p-1} 3^{i-1}\left\{\widetilde{H}\left[\sum_{j=0}^{\infty} G^{[2 j+1]}(y), \sum_{j=0}^{\infty} G^{[2 j+1]}(y), \cdot\right]\right\}^{i-2}\left[\sum_{k=0}^{\infty} G^{[2 k+1]}(y)-y\right],
\end{aligned}
$$

where the power series mapping $R_{1} \geq 0$. Repeating the procedure leading from (9) to (12), we get in the end

$$
\sum_{j=0}^{\infty}\left(2 j+1-3-2 \cdot 3^{1}-\ldots-2 \cdot 3^{p-2}\right) G^{[2 j+1]}(y)+R(y)=0
$$


i.e.

$$
\sum_{j=0}^{\infty}\left(2 j+1-3^{p-1}\right) G^{[2 j+1]}(y)+R(y)=0,
$$

with some formal power series mapping $R=\sum_{m=0}^{\infty} R^{[m]} \geq 0$. Hence, for every $j \in \mathbb{N}$,

$$
\left(2 j+1-3^{p-1}\right) G^{[2 j+1]}(y)+R^{[2 j+1]}(y)=0, \quad R^{[2 j+1]} \geq 0 .
$$

By (10) and (13) we have $G^{[2 j+1]}=0$ for $2 j+1>3^{p-1}$.

Remark 3.4. Let $\mathbb{R}^{(\mathbb{N})}$ denote the direct sum of $\mathbb{R}$ over $\mathbb{N}$. We call $G=$ $\left(G_{1}, G_{2}, \ldots\right)$ a polynomial automorphism of $\mathbb{R}^{(\mathbb{N})}$ if there exists a natural number $p$ such that the mapping $\left(G_{1}, \ldots, G_{p}\right)$ is a polynomial automorphism of $\mathbb{R}^{p}$ and $G_{i}\left(x_{1}, x_{2}, \ldots\right)=x_{i}$ when $i>p$; cf. [D2]. Since we do not need to use the dimension of the space $\mathbb{R}^{n}$ in the proof of Theorem 3.3, it remains true for the polynomial mappings of $\mathbb{R}^{(\mathbb{N})}$.

Added in proof. Unfortunately, the inequality

$$
\operatorname{deg} F^{-1} \leq(\operatorname{deg} F)^{\text {ind } F-1}
$$

conjectured in $[\mathrm{DR}]$ and partially confirmed by Theorem 3.3 is not true in the general case, as has been shown recently by A. van den Essen in [E3].

\section{References}

[BCW] H. Bass, E. H. Connell and D. Wright, The Jacobian Conjecture: reduction of degree and formal expansion of the inverse, Bull. Amer. Math. Soc. 7 (1982), $287-330$.

[BR] A. Białynicki-Birula and M. Rosenlicht, Injective morphisms of real algebraic varieties, Proc. Amer. Math. Soc. 13 (1962), 200-203.

[D1] L. M. Drużkowski, An effective approach to Keller's Jacobian Conjecture, Math. Ann. 264 (1983), 303-313.

[D2] - The Jacobian Conjecture in case of rank or corank less than three, J. Pure Appl. Algebra 85 (1993), 233-244.

[D3] - , The Jacobian Conjecture, preprint 492, Institute of Mathematics, Polish Academy of Sciences, Warszawa, 1991.

[D4] - The Jacobian Conjecture: some steps towards solution, in: Automorphisms of Affine Spaces, A. van den Essen (ed.), Kluwer, 1995, 41-53.

[DR] L. M. Drużkowski and K. Rusek, The formal inverse and the Jacobian Conjecture, Ann. Polon. Math. 46 (1985), 85-90.

[E1] A. van den Essen, Polynomial maps and the Jacobian Conjecture, Report 9034, Catholic University, Nijmegen, 1990.

[E2] - The exotic world of invertible polynomial maps, Nieuw Arch. Wisk. (4) 11 (1) (1993), 21-31.

[E3] - A counterexample to a conjecture of Drużkowski and Rusek, Ann. Polon. Math. 62 (1995), 173-176. 
[K] O.-H. Keller, Ganze Cremona-Transformationen, Monatsh. Math. Phys. 47 (1939), 299-306.

[KS] T. Krasiński and S. Spodzieja, On linear differential operators related to the n-dimensional Jacobian Conjecture, in: Real Algebraic Geometry, M. Coste, L. Mahé and M.-F. Roy (eds.), Lecture Notes in Math. 1524, Springer, 1992, 308-315.

[M] G. H. Meisters, Jacobian problems in differential equations and algebraic geometry, Rocky Mountain J. Math. 12 (1982), 679-705.

[MO] G. H. Meisters and C. Olech, A poly-flow formulation of the Jacobian Conjecture, Bull. Polish Acad. Sci. Math. 35 (1987), 725-731.

[P] S. Pinchuk, A counterexample to the real Jacobian Conjecture, Math. Z. 217 (1994), 1-4.

[R] K. Rusek, Polynomial automorphisms, preprint 456, Institute of Mathematics, Polish Academy of Sciences, Warszawa, 1989.

[RW] K. Rusek and T. Winiarski, Polynomial automorphisms of $\boldsymbol{C}^{n}$, Univ. Iagel. Acta Math. 24 (1984), 143-149.

[S] Y. Stein, The Jacobian problem as a system of ordinary differential equations, Israel J. Math. 89 (1995), 301-319.

[W] T. Winiarski, Inverse of polynomial automorphisms of $\mathbf{C}^{n}$, Bull. Acad. Polon. Sci. Sér. Sci. Math. 27 (1979), 673-674.

[Y] A. V. Yagzhev, On Keller's problem, Sibirsk. Mat. Zh. 21 (1980), 141-150 (in Russian).

[Yu] J.-T. Yu, On the Jacobian Conjecture: reduction of coefficients, J. Algebra 171 (1995), 515-523.

Institute of Mathematics

Jagiellonian University

Reymonta 4/508

30-059 Kraków, Poland

E-mail: druzkows@im.uj.edu.pl

Reçu par la Rédaction le 30.7.1995 